\title{
HARMONIC MAPS AND A PINCHING THEOREM FOR POSITIVELY CURVED HYPERSURFACES
}

\author{
H. S. HU, Y. L. PAN AND Y. B. SHEN
}

\begin{abstract}
In this paper, we establish a theorem of Liouville type for stable harmonic maps in sufficiently pinched, positively curved hypersurfaces of a space form with nonnegative constant curvature. Similar results for the Euclidean sphere $S^{n}$ have been proved by Y. L. Xin and P. F. Leung, respectively.
\end{abstract}

1. Statement of result. As is well known, for the Euclidean $n(\geq 3)$-sphere $S^{n}$, there are no nonconstant stable harmonic maps either from $S^{n}$ to any Riemannian manifold [6] or from any compact manifold to $S^{n}$ [3]. Its generalizations to submanifolds have been attempted by many authors $[\mathbf{3}, \mathbf{4}, \mathbf{5}]$, where the additional conditions imposed on submanifolds are extrinsic. It is natural to find some condition intrinsic for submanifolds. In this paper, we prove the following theorem.

THEOREM. Let $\tilde{M}^{n+1}(c)$ be an $(n+1)$-dimensional simply connected space form with constant sectional curvature $c \geq 0$. Suppose that $M^{n}(n \geq 3)$ is a compact hypersurface in $\tilde{M}^{n+1}(c)$ of which the sectional curvature Riem ${ }^{M}$ satisfies the following pinching condition:

$$
c+a^{2} /[(n-2) c+(n-1) a] \leq \operatorname{Riem}^{M}<c+a
$$

for some constant $a>0$. Then there is no nonconstant stable harmonic map between $M^{n}$ and any compact Riemannian manifold $N$, i.e., either from $M^{n}$ to $N$ or from $N$ to $M^{n}$.

COROLLARY 1. If $M^{n}(n \geq 3)$ is a compact hypersurface in the Euclidean space $E^{n+1}$ with pinching condition: $a /(n-1) \leq \operatorname{Riem}^{M}<a$ for some constant $a>0$, then there is no nonconstant stable harmonic map between $M^{n}$ and any compact Riemannian manifold.

COROLLARY 2. If $M^{n}(n>4)$ is a compact hypersurface in $E^{n+1}$ with $\frac{1}{4} \leq$ Riem $^{M}<1$, then the same conclusion as in Corollary 1 holds.

REMARK. The condition (1) is sharp in some sense; see an example below.

2. Preliminaries. Let $M$ and $N$ be Riemannian manifolds of dimensions $n$ and $m$, respectively, and $\phi: M \rightarrow N$ a smooth map. Throughout this paper, we agree on the following ranges of indices unless otherwise stated:

$$
1 \leq i, j, k, \ldots \leq n ; \quad 1 \leq \alpha, \beta, \gamma, \ldots \leq m
$$

Received by the editors October $1,1985$.

1980 Mathematics Subject Classification (1985 Revision). Primary 58E20; Secondary 53C40.

Key words and phrases. Harmonic map, hypersurface, stable, pinching theorem. 
We choose local fields of orthonormal frames $\left\{e_{i}\right\}$ and $\left\{e_{\alpha}^{\prime}\right\}$ in $M$ and $N$, respectively, and let $\left\{\omega_{i}\right\}$ and $\left\{\omega_{\alpha}^{\prime}\right\}$ be the fields of dual frames. Under the map $\phi$, we have $\phi^{*} \omega_{\alpha}^{\prime}=\sum_{i} a_{\alpha i} \omega_{i}$. The energy of $\phi$ is defined by

$$
E(\phi)=\frac{1}{2} \int_{M} \sum_{\alpha, i}\left(a_{\alpha i}\right)^{2} * 1,
$$

where $^{*} 1=\omega_{1} \wedge \cdots \wedge \omega_{n}$. The map $\phi$ is harmonic iff its tension field $\tau=\sum_{\alpha, i} a_{\alpha i i} e_{\alpha}^{\prime}$ vanishes identically, where $a_{\alpha i j}$ is the covariant derivative of $a_{\alpha i}[\mathbf{1}]$.

For any deformation vector field along $\phi, V=\sum_{\alpha} v_{\alpha} e_{\alpha}^{\prime}$, the second variation of $E(\phi)$ is $[\mathbf{2}, \mathbf{4}]$

$$
E^{\prime \prime}(\phi)=-\int_{M}\left[\sum_{\alpha} v_{\alpha}\left(\triangle_{M} v_{\alpha}+\sum_{i, \beta, \gamma, \delta} v_{\delta} a_{\beta i} a_{\gamma i} R_{\beta \alpha \gamma \delta}^{\prime}\right)\right] * 1,
$$

where $R_{\alpha \beta \gamma \delta}^{\prime}$ is the curvature tensor of $N$ and $\Delta_{M}$ is the Laplacian on $M$. If $E^{\prime \prime}(\phi) \geq 0$ for any $V$ along $\phi$, then the harmonic map $\phi$ is said to be stable.

Now let $\tilde{M}^{n+1}(c)$ be an $(n+1)$-dimensional simply connected space form with constant sectional curvature $c \geq 0$, and $M^{n}$ a hypersurface in $\tilde{M}^{n+1}(c)$. We choose a local field of orthonormal frames $e_{1}, \ldots, e_{n+1}$ in $\tilde{M}^{n+1}(c)$ such that, restricted to $M^{n}$, the vector $e_{n+1}$ is normal to $M^{n}$. Let $\omega_{1}, \ldots, \omega_{n+1}$ be the field of dual frames relative to the frame field chosen above. Then, restricted to $M$, we have [1]

$$
\omega_{n+1}=0, \quad \omega_{n+1, i}=\sum_{j} h_{i j} \omega_{j}, \quad h_{i j}=h_{j i} .
$$

The Gauss equation of $M^{n}$ in $\tilde{M}^{n+1}(c)$ is

$$
R_{i j k l}=c\left(\delta_{i k} \delta_{j l}-\delta_{i l} \delta_{j k}\right)+h_{i k} h_{j l}-h_{i l} h_{j k},
$$

where $R_{i j k l}$ is the curvature tensor of $M^{n}$.

By [4 and 5], we have the following lemma, whose proof is omitted here.

LEMMA 1. Let $M^{n}(n \geq 3)$ be a compact hypersurface in $\tilde{M}^{n+1}(c)$ with $c \geq 0$. If the second fundamental tensor $h_{i j}$ of $M^{n}$ satisfies pointwisely

$$
\sum_{k}\left(2 h_{i k} h_{j k}-h_{k k} h_{i j}\right)<(n-2) c \delta_{i j}
$$

for an orthonormal basis $\left\{e_{i}\right\}$ on $M^{n}$, then there is no nonconstant stable harmonic map between $M^{n}$ and any compact Riemannian manifold.

3. The proof of the Theorem. Let $P$ be an arbitrary point in $M^{n}$ and $\left\{\lambda_{i}\right\}$ eigenvalues of the matrix $\left(h_{i j}\right)$. It follows from (3) that

$$
R_{i j i j}=c+\lambda_{i} \lambda_{j} \quad(i \neq j)
$$

at $P \in M$. The pinching condition (1) together with (5) implies that all of $\left\{\lambda_{i}\right\}$ are nonzero and have the same sign. So, without loss of generality, we may assume that at $P$

$$
0<\lambda_{i} \leq \lambda_{2} \leq \cdots \leq \lambda_{n}
$$


and set

$$
H=\sum_{i} \lambda_{i}
$$

Thus, (4) becomes

$$
2 \lambda_{i}^{2}-H \lambda_{i}-(n-2) c<0
$$

for all $i$, which together with (6) yields that

$$
\lambda_{i} \leq \lambda_{n}<\frac{1}{4}\left[H+\sqrt{H^{2}+8(n-2) c}\right] .
$$

Hence, we have the following lemma.

LEMMA 2. Under the assumption (6), the condition (4) is equivalent to

$$
\lambda_{n}<\frac{1}{4}\left[H+\sqrt{H^{2}+8(n-2) c}\right]
$$

at $P \in M$.

We now suppose that

$$
b^{2} \leq \lambda_{i} \lambda_{j}<B^{2} \quad(i \neq j)
$$

for some $0<b<B$ at $P \in M$.

LEMMA 3. If $\lambda_{1} \geq b$ and $B, b$ in (9) satisfy

$$
B^{2}-L b+(n-1) b^{2}=0,
$$

where

$$
L=\frac{1}{2}\left[3(n-1) b+\sqrt{(n-1)^{2} b^{2}+4(n-2) c}\right],
$$

then the inequality (8) holds.

ProOF. Since $\lambda_{1} \geq b$, it follows from (9) and (10) that

$$
\lambda_{n}<B^{2} / \lambda_{1} \leq B^{2} / b=L-(n-1) b .
$$

On the other hand, we have from (11)

$$
[3 L-4(n-1) b]^{2}=L^{2}+8(n-2) c,
$$

i.e.,

$$
L-(n-1) b=\frac{1}{4}\left[L+\sqrt{L^{2}+8(n-2) c}\right] .
$$

Substituting (13) into (12), we get

$$
\lambda_{n}<\frac{1}{4}\left[L+\sqrt{L^{2}+8(n-2) c}\right] .
$$

If $H \geq L,(14)$ implies (8). So we need only consider the case that $H<L$. Putting

$$
L-H=K>0,
$$

we see from (6), (7) and (15)

$$
\lambda_{n}=H-\sum_{j \neq n} \lambda_{j} \leq H-(n-1) b=L-(n-1) b-K,
$$


which together with (13) yields

$$
\lambda_{n} \leq \frac{1}{4}\left[H+\sqrt{L^{2}+8(n-2) c}-3 K\right] .
$$

On the other hand, from the fact that $K>0$ and $c \geq 0$ it is easy to see that

$$
\left[\sqrt{(L-K)^{2}+8(n-2) c}+3 K\right]^{2}>L^{2}+8(n-2) c
$$

i.e.,

$$
\sqrt{H^{2}-8(n-2) c}>\sqrt{L^{2}+8(n-2) c}-3 K .
$$

Substituting (17) into (16), we complete the proof of Lemma 3.

LEMMA 4. If $n \geq 3$, and (10) holds, then (8) is true.

Proof. By Lemma 3, we need only consider the case that $\lambda_{1}<b$. Since $n \geq 3$, there exist $\lambda_{1}$ and $\lambda_{2}\left(\leq \lambda_{3}\right)$ such that, by (9), $\lambda_{1} \lambda_{2} \geq b^{2}$.

Construct

$$
\lambda_{1}^{\prime}=\lambda_{2}^{\prime}=\frac{1}{2}\left(\lambda_{1}+\lambda_{2}\right)
$$

Then,

$$
\left(\lambda_{1}^{\prime}\right)^{2}=\frac{1}{4}\left(\lambda_{1}+\lambda_{2}\right)^{2} \geq \lambda_{1} \lambda_{2} \geq b^{2}
$$

so that $\lambda_{1}^{\prime} \geq b$. Since (18) implies that $\lambda_{1}^{\prime}=\lambda_{2}^{\prime} \leq \lambda_{3}$ and $\lambda_{1}^{\prime}+\lambda_{2}^{\prime}+\sum_{k=3}^{n} \lambda_{k}=H$, then we can apply Lemma 3 to the case where $0<\lambda_{1}^{\prime}=\lambda_{2}^{\prime} \leq \lambda_{3} \leq \cdots \leq \lambda_{n}$ and conclude that (8) is true. Thus, Lemma 4 is proved completely.

Now, we can easily give the proof of the Theorem.

It is clear, by (5), that (1) becomes

$$
a^{2} /[(n-2) c+(n-1) a] \leq \lambda_{i} \lambda_{j}<a \quad(i \neq j)
$$

at $P \in M$.

Taking $b=a / \sqrt{(n-2) c+(n-1) a}$, we have

$$
a=\frac{1}{2} b\left[(n-1) b+\sqrt{(n-1)^{2} b^{2}+4(n-2) c}\right] .
$$

Combining (19) with (20), we obtain (9) and (10) at $P$. By Lemmas 2 and 3, we conclude that (4) holds at $P$. Since $P \in M$ is arbitrary, the Theorem follows immediately from Lemma 1.

EXAMPLE. In order to employ Lemma 1, the inequalilty $\operatorname{Riem}^{M}<c+a$ in (1) cannot be replaced by $\operatorname{Riem}^{M} \leq c+a$. In such a sense, our pinching condition (4) is sharp. In fact, we may set

$$
\lambda_{1}=\cdots=\lambda_{n-1}=b, \quad \lambda_{n}=\frac{1}{2}\left[(n-1) b+\sqrt{(n-1)^{2} b^{2}+4(n-2) c}\right]
$$

where

$$
b=a / \sqrt{(n-2) c+(n-1) a}
$$

for some constant $a>0$. In this case, we have (6) and

$$
b^{2} \leq \lambda_{i} \lambda_{j} \leq \frac{1}{2} b\left[(n-1) b+\sqrt{(n-1)^{2} b^{2}+4(n-2) c}\right] \quad(i \neq j)
$$

which together with (5) implies that

$$
c+a^{2} /[(n-2) c+(n-1) a] \leq \operatorname{Riem}^{M} \leq c+a .
$$


On the other hand, from (21) and (11) we can see that

$$
H=(n-1) b+\lambda_{n}=\frac{1}{2}\left[3(n-1) b+\sqrt{(n-1)^{2} b^{2}+4(n-2) c}\right]=L,
$$

which together with (13) yields that

$$
\lambda_{n}=\frac{1}{4}\left[H+\sqrt{H^{2}+8(n-2) c}\right],
$$

i.e., (8) is not true. Thus, Lemma 1 cannot be applied to this case.

ACKNOWLEDGEMENT. The third-named author would like to thank Professor S. S. Chern and the Mathematical Sciences Research Institute for their hospitality.

\section{REFERENCES}

1. S. S. Chern and S. I. Goldberg, On the volume decreasing property of a class of real harmonic mappings, Amer. J. Math. 97 (1975), 133-147.

2. J. Eells and L. Lemaire, A report on harmonic maps, Bull. London Math. Soc. 10 (1978), 1-68.

3. P. F. Leung, On the stability of harmonic maps, Lecture Notes in Math., vol. 949, SpringerVerlag, pp. 122-129.

4. Y. L. Pan and Y. B. Shen, Stability of harmonic maps and minimal immersions, Proc. Amer. Math. Soc. 93 (1985), 111-117.

5. Y. L. Xin, Topology of certain submanifolds in the Euclidean sphere, Proc. Amer. Math. Soc. 82 (1981), 643-648.

6. __ Some results on stable harmonic maps, Duke Math. J. 47 (1980), 609-613.

Department of Mathematics, Fudan University, Shanghai, People's RepubLIC OF CHINA (Current address of H. S. Hu and Y. L. Pan)

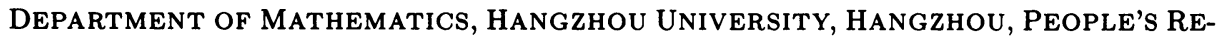
PUBLIC OF CHINA (Current address of Y. B. Shen) 